OPEN ACCESS

Edited by:

Xiaoxing Xiong,

Wuhan University, China

Reviewed by:

Jiping Tang,

Loma Linda University, United States

Yanqin Gao

Fudan University, China

${ }^{*}$ Correspondence:

Jieli Chen

jchen4@hfhs.org

Specialty section:

This article was submitted to

Multiple Sclerosis and

Neuroimmunology,

a section of the journal

Frontiers in Immunology

Received: 18 September 2019 Accepted: 08 November 2019 Published: 26 November 2019

Citation:

Chen Z, Chopp M, Zacharek A, Li W, Venkat $P$, Wang F, Landschoot-Ward J and Chen J (2019) Brain-Derived Microparticles (BDMPs) Contribute to Neuroinflammation and Lactadherin Reduces BDMP Induced Neuroinflammation and Improves Outcome After Stroke.

Front. Immunol. 10:2747. doi: 10.3389/fimmu.2019.02747

\section{Brain-Derived Microparticles (BDMPs) Contribute to Neuroinflammation and Lactadherin Reduces BDMP Induced Neuroinflammation and Improves Outcome After Stroke}

\author{
Zhili Chen ${ }^{1}$, Michael Chopp ${ }^{1,2}$, Alex Zacharek ${ }^{1}$, Wei Li ${ }^{1}$, Poornima Venkat ${ }^{1}$, Fenjie Wang ${ }^{1}$, \\ Julie Landschoot-Ward ${ }^{1}$ and Jieli Chen ${ }^{1 *}$
}

${ }^{1}$ Department of Neurology, Henry Ford Hospital, Detroit, MI, United States, ${ }^{2}$ Department of Physics, Oakland University, Rochester, MI, United States

Microparticles (MPs, size between 0.1 and $1 \mathrm{~mm}$ ) are lipid encased containers derived from intact cells which contain antigen from the parent cells. MPs are involved in intercellular communication and regulate inflammation. Stroke increases secretion of brain derived MP (BDMP) which activate macrophages/microglia and induce neuroinflammation. Lactadherin (Milk fat globule-EGF factor-8) binds to anionic phospholipids and extracellular matrices, promotes apoptotic cell clearance and limits pathogenic antigen cross presentation. In this study, we investigate whether BDMP affects stroke-induced neuroinflammation and whether Lactadherin treatment reduces stroke initiated BDMP-induced neuroinflammation, thereby improving functional outcome after stroke. Middle aged (8-9 months old) male C57BL/6J mice were subjected to distal middle cerebral artery occlusion (dMCAo) stroke, and BDMPs were extracted from ischemic brain $24 \mathrm{~h}$ after dMCAo by ultracentrifugation. Adult male C57BL/6J mice were subjected to $\mathrm{dMCAo}$ and treated via tail vein injection at $3 \mathrm{~h}$ after stroke with: $(\mathrm{A})$

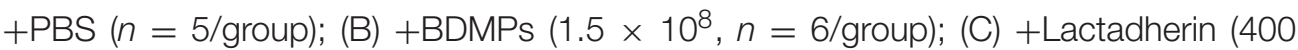
$\mu \mathrm{g} / \mathrm{kg}, n=5 /$ group); (D) $+B D M P+$ Lactadherin ( $n=6 /$ group). A battery of neurological function tests were performed and mice sacrificed for immunostaining at 14 days after stroke. Blood plasma was used for Western blot assay. Our data indicate: (1) treatment of Stroke with BDMP significantly increases lesion volume, neurological deficits, blood brain barrier (BBB) leakage, microglial activation, inflammatory cell infiltration (CD45, microglia/macrophages, and neutrophils) into brain, inflammatory factor (TNF $\alpha$, IL6, and IL1 $\beta$ ) expression in brain, increases axon/white matter (WM) damage identified by decreased axon and myelin density, and increases inflammatory factor expression in the plasma when compared to PBS treated stroke mice; (2) when compared to PBS and 
BDMP treated stroke mice, Lactadherin and BDMP+Lactadherin treatment significantly improves neurological outcome, and decreases lesion volume, BBB leakage, axon/WM injury, inflammatory cell infiltration and inflammatory factor expression in the ischemic brain, respectively. Lactadherin treatment significantly increases anti-inflammatory factor (IL10) expression in ischemic brain and decreases IL1 $\beta$ expression in plasma compared to PBS and BDMP treated stroke mice, respectively. BDMP increases neuroinflammation and aggravates ischemic brain damage after stroke. Thus, Lactadherin exerts anti-inflammatory effects and improves the clearance of MPs to reduce stroke and BDMP induced neurological deficits.

Keywords: brain-derived microparticles, stroke, neuroinflammation, Lactadherin, therapy

\section{INTRODUCTION}

Stroke is a leading cause of mortality and severe long-term disability worldwide (1). In addition to the challenges of dayto-day activities due to neurological deficits, stroke is also a huge economic burden to patients and caregivers (2). Despite extensive research in the past few decades, there are few treatment options for stroke and stroke remains a global health concern (3). Therefore, there is an urgent need to identify effective treatments for stroke.

Microparticles (MPs, size between 0.1 and $1 \mathrm{~mm}$ ) are a class of small membrane-bound vesicles that are shed from the cell membrane (4). MPs can be released into the blood and body fluids by cells during activation, necrosis or apoptosis (5). A massive amount of MPs are released into the circulation after acute brain injury (6-9). These MPs are broadly classified into: (1) circulating MPs that are derived from endothelial cells, platelets, leukocytes, and (2) brain derived MPs (BDMPs) (10, 11). Both types of MPs may be involved in disease development. Circulating MPs have been investigated as potential biomarkers for a variety of neurological disorders including ischemic cerebrovascular accidents, transient ischemic attacks, multiple sclerosis, and cerebral malaria (12). MPs play a key role in peripheral inflammatory progression, thrombosis, endothelial dysfunction, and angiogenesis (13-15). Microglial/macrophagederived MPs and BDMPs can increase brain inflammation in normal mice $(16,17)$. MPs increase the permeability of bloodbrain barrier (BBB) (18) and BDMPs can migrate through the disrupted endothelial barrier (19). These observations led us to hypothesize that BDMPs contribute to neuroinflammation after stroke, and thereby increased BDMP clearance would improve neurological recovery.

Lactadherin (milk fat globule-epidermal growth factor 8, Lactadherin) is a multifunctional glycoprotein originally identified as part of the milk fat globule membrane. Lactadherin couples apoptotic cells with monocytes/macrophages to facilitate phagocytosis (20-22) and clearance of apoptotic cells, and regulates immune response after stroke (23-26). In this study, we are the first to investigate that BDMPs contribute to neuroinflammation after stroke, while Lactadherin promotes the clearance of BDMPs and reduces inflammation and thereby improves ischemic stroke outcome.

\section{MATERIALS AND METHODS}

All experiments were conducted in accordance with the standard and procedures of the American Council on Animal Care and Institutional Animal Care and Use Committee of Henry Ford Health System.

\section{Experimental Groups}

Middle aged (8-9 months) male C57/BL6 mice (Jackson Laboratory) were subjected to distal middle cerebral artery occlusion (dMCAo) and randomly divided into the following treatment groups: (1) PBS $(n=5)$; $(2)+$ BDMPs $\left(1.5 \times 10^{8}\right.$, $n=6)$; (3) +Lactadherin $(400 \mu \mathrm{g} / \mathrm{kg}$, tail vein injection, Hematologic Technologies, Essex Junction, VT, $n=5$ ); (4) +BDMP+Lactadherin $(n=6)$; (5) Sham control $(n=6)$; (6) Sham+BDMP $(n=5)$. Treatments were administered via tail vein injection at $3 \mathrm{~h}$ after stroke.

\section{Photothrombotic Stroke Model}

To generate a consistent infarct volume, focal cortical ischemia was induced by photothrombosis of the cortical microvessels, as previously described (27). Briefly, mice were anesthetized with chloral hydrate $(0.3 \mathrm{mg} / \mathrm{kg}$, i.p). A light sensitive dye, Rose Bengal $(100 \mu \mathrm{l} /<25 \mathrm{~g}, 150 \mu \mathrm{l} / 25-40 \mathrm{~g}, 10 \mathrm{mg} / \mathrm{ml}$ solution in saline; SigmaAldrich, St Louis, MO) was administered i.p. A midline incision of the scalp was performed to expose the skull. The skull was covered by a roundabout black rubber to expose the area of $0.7-2.7 \mathrm{~mm}$ right to the midline, $-2.5-1 \mathrm{~mm}$ rostral to the bregma. The brain was illuminated for $15 \mathrm{~min}$ through the exposed skull with a fiber-optic bundle of a cold light source (KL 1600 LED; Schott, Mainz, Germany) filtered with a green filter. The scalp incision was sutured and mice returned to home cages to awaken. Sham control mice were subjected to the same surgical protocol as above, but without injection of Rose Bengal.

\section{Neurological Function Test}

To assess neurological functional outcome, a battery of functional tests including a modified neurological severity score (mNSS) test (28) and foot-fault test (29) were performed before dMCAo and after dMCAo on days 1, 3, 7 and 14 by an investigator who was blinded to the experimental groups. 


\section{BDMP Isolation}

BDMP isolation was performed following previously published methods $(7,9,19)$. Briefly, the ischemic brain was harvested $24 \mathrm{~h}$ after stroke and quickly frozen in liquid nitrogen. To isolate BDMPs, brain was rapidly thawed at room temperature and homogenized in $1 \mathrm{ml}$ of PBS using a glass Dounce homogenizer (Fisher Scientific Co., Federal Way, WA). The homogenate was centrifuged at $1,500 \mathrm{~g}$ for $20 \mathrm{~min}$ at $4^{\circ} \mathrm{C}$ to remove intact cells. The supernatant was centrifuged at $13,000 \mathrm{~g}$ for $2 \mathrm{~min}$ at $4^{\circ} \mathrm{C}$ to remove large cellular debris, and then centrifuged twice at $100,000 \mathrm{~g}$ for $1 \mathrm{~h}$ at $4^{\circ} \mathrm{C}$, using a TLA-100.4 rotor (Beckman Coulter, Miami, FL). The pellet was resuspended in $500 \mu$ l of PBS. MPs were quantified by flow cytometry in a time fixed mode in the presence of counting beads (Spherotech, Lake Forest, IL). Megamix microbeads [0.5, 0.9 and $3 \mu \mathrm{m}$ (Biocytex, Marseille, France)] were used to gate microparticles based on the particle size.

\section{Immunohistochemistry}

All animals were euthanized 14 days after stroke and transcardially perfused with cold $0.9 \%$ saline. Brains were isolated and immersion fixed in $4 \%$ paraformaldehyde before being embedded in paraffin. A series brain coronal sections $(6 \mu \mathrm{m}$ thick) were cut from the center of the lesion (bregma $-2.5 \mathrm{~mm} \sim+1 \mathrm{~mm})$. Hematoxylin \& eosin ( $\mathrm{H} \& \mathrm{E})$ stain was used to identify the lesion volume. All immunostainings were performed at 14 days after stroke. Antibodies against CD45 (a marker for lymphocytes, 1:500, Abcam), IBA-1 (a marker for microglia/macrophages, 1:1,000, Abcam), myelin basic protein (MBP, a marker for myelin, 1:300, Dako), CD31 (a marker for vessel, 1:200, Dako), Antibody against albumin (AlbuminFITC, 1:500, Abcam), NeuN (a marker for neuronal,1:50, Millepore), Myelo-peroxidase (MPO, a marker for neutrophil, 1:200, Dako), Interleukin $1 \beta$ (IL1 $\beta, 1: 200$, Abcam), Interleukin 6 (IL6,1:200, Abcam), Tumor necrosis factor (TNF $\alpha, 1 ; 200$, Abcam), and Interleukin 10 (IL10, 1:200, Abcam) were employed. Bielschowsky silver (BS) staining was used to demonstrate axons and luxol fast blue (LFB) staining was used to demonstrate myelin. Three slides from each brain, with each slide containing five fields from cortex and striatum of the ischemic border zone (IBZ) were digitized under $20 \times$ objective (Olympus BX40) using a microscope (Sony DXC-970MD). The number of positive cells of neuronal, CD31, CD45, IBA-1, neutrophils, IL6, IL1 $\beta$, TNF $\alpha$, IL10, and the positive areas of Albumin, BS, LFB and MBP were calculated by Image Pro Plus 6.0. Immunohistochemical analysis was performed by an investigator who was blinded to the experimental groups.

\section{Lesion Volume Measurement}

Seven coronal sections of tissue were stained with hematoxylin and eosin (H\&E) for lesion volume calculation. Data are presented as a percentage of lesion compared with the contralateral hemisphere (30). Measurements were performed by an investigator who was blinded to the experimental groups.

\section{Western Blot}

Equal amounts of plasma samples were subjected to Western blot analysis, as previously described (31). Protein concentration was measured using BCA Protein Assay Kit (Thermo Fisher Scientific, USA). Forty micrograms of protein/lane in a $10 \%$ SDS PAGE precast gel (Invitrogen). Gel was transferred using an iBlot transfer system (Invitrogen) following standard protocol. Nitrocellulose membrane was blocked in 2\% I-Block (Applied Biosystems) in $1 \times$ TBS-T for $1 \mathrm{~h}$. Primary antibody against IL1 $\beta$ (1:1,000, Abcam, Cambridge, MA, USA) was employed. Anti- $\beta$-actin (1:10,000, Abcam, Cambridge, MA, USA) was employed for control measurements. Secondary antibody was added at 1:5,000 dilution in 2\% I-Block in $1 \times$ TBS-T on a room temperature shaker for $1 \mathrm{~h}$. The membranes were then developed using a FluorChem E Imager system (ProteinSimple) exposing them for 1-30 min depending on the intensity of the band. Bands were analyzed using ImageJ.

\section{Statistical Analysis}

Repeated measure analysis of variance (ANCOVA) was used to study the group differences in mNSS and foot-fault function tests over time (time points: 1, 3, 7, and 14 days). The one-way analysis of variance (ANOVA) was used to evaluate immunostaining and Western blot. All data are presented as mean \pm SE.

\section{RESULTS}

\section{BDMPs Do Not Induce Neurological Deficit and Brain Damage in Wild Type Control Mice}

First, we tested whether BDMPs induce brain damage and neurological function deficit in non-stroke sham control mice. We found that (Supplementary Figure 1) injection of BDMPs into sham non-stroke (Sham+BDMP) mice did not induce neurological functional deficits (Supplementary Figures 1A,B), and no axon/white matter damage was evident in the brain tissue identified by BS and LFB staining when compared to sham control mice (Supplementary Figures 1C,D). Injection of BDMP into sham non-stroke (Sham+BDMP) mice did not induce leukocyte (CD45, Supplementary Figure 1E) infiltration or increase microglial activation (IBA-1, Supplementary Figure 1F) in brain when compared to sham control mice.

\section{BDMPs Aggravate and Lactadherin Treatment Attenuates Neurological Impairment and Lesion Volume After Stroke in Mice}

To evaluate the effects of BDMPs and Lactadherin treatment on neurological function after stroke in mice, mNSS, and footfault tests were employed. Figures 1A,B shows that injection of BDMPs significantly aggravates neurological impairment in stroke mice when compared to PBS dMCAo control group. Injection of BDMPs significantly increases ischemic lesion 

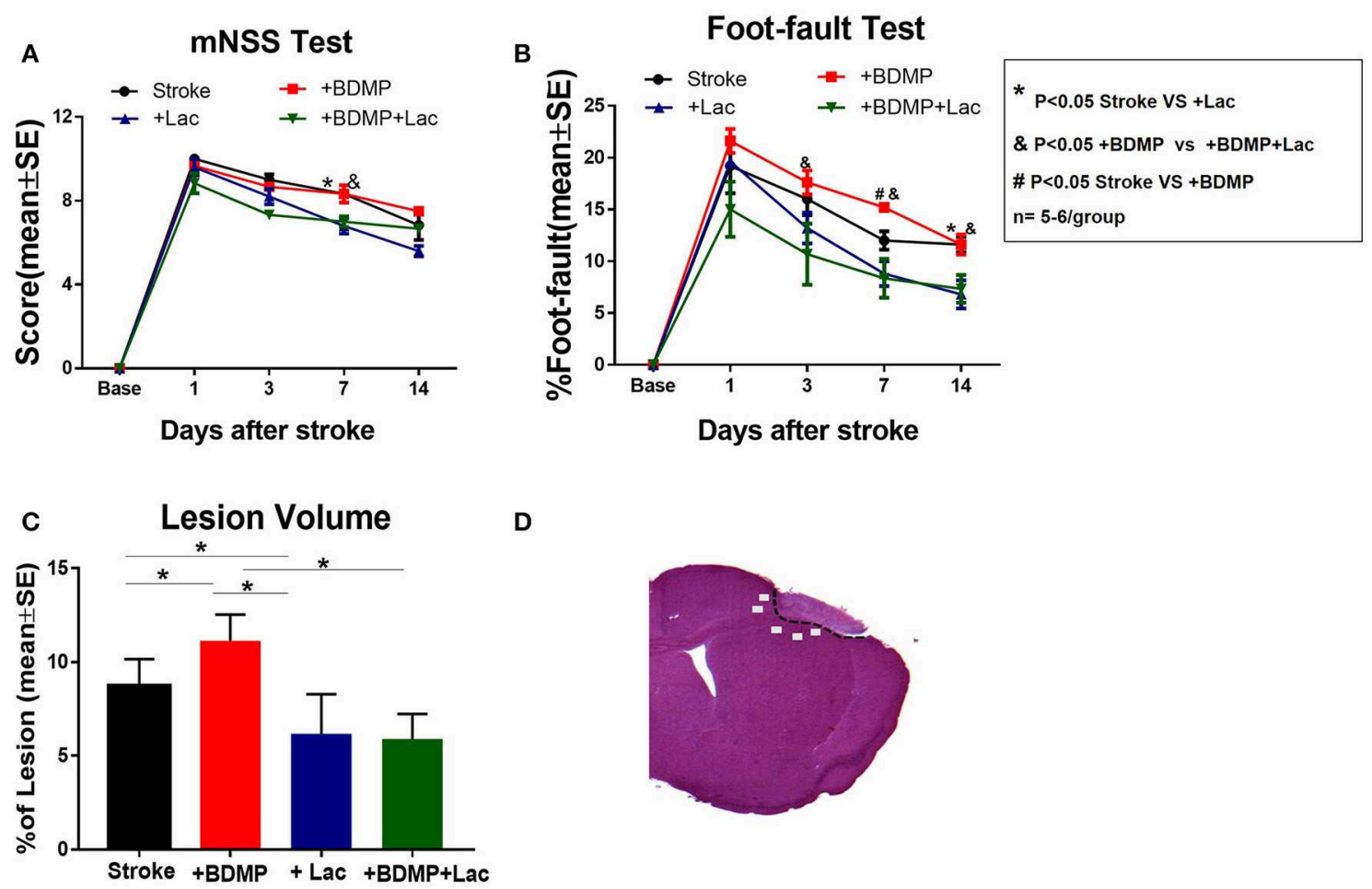

D

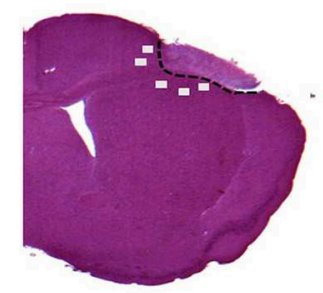

FIGURE 1 | BDMPs aggravate and Lactadherin attenuates neurological impairment and lesion volume after stroke in mice. (A) mNSS and (B) Foot-fault tests were performed at 1, 3, 7, and 14 days after stroke. (C) Stroke lesion volume was calculated by H\&E stains. $n=5-6 /$ group. (D) Immunostaining measurement from cortex and striatum of the selected five fields in the ischemic border zone (IBZ). ${ }^{\star} P<0.05$ Stroke vs. Stroke+lac; $\& P<0.05$ Stroke+BDMP vs. Stroke+BDMP+lac; \# $P<$ 0.05 Stroke vs. Stroke+BDMP; $n=5-6 /$ group; Data are presented as mean \pm SE.

volume in the brain identified by H\&E staining (Figure 1C). Administration of Lactadherin together with PBS or BDMPs significantly improves neurological function when compared to stroke mice receiving $\mathrm{PBS}$ or BDMPs alone, respectively (Figures 1A,B). Administration of Lactadherin together with PBS or BDMPs significantly decreases ischemic lesion volume when compared to stroke mice receiving PBS or BDMPs alone, respectively (Figure 1C).

\section{BDMP Aggravates and Lactadherin Attenuates BBB Leakage and Neuronal Loss While Increasing Vascular Density in Stroke Mice}

To evaluate the effects of circulating BDMPs and Lactadherin on BBB integrity, vasculature and neuronal injury, we evaluated FITC-albumin, neuron and vascular density in the cortex and striatum of IBZ (Figure 1D). Figure 2 shows that BDMPs injection significantly increases $\mathrm{BBB}$ permeability, decreases vascular density and results in greater neuronal loss compared to PBS treated stroke mice. Lactadherin treatment significantly decreases BBB leakage, increases vascular density and attenuates neuronal loss compared to stroke mice treated with PBS or BDMPs, respectively (Figure 2).

\section{BDMPs Significantly Increase Axonal/WM Damage in Ischemic Brain While Lactadherin Treatment Significantly Promotes Axonal/WM Density After Stroke in Mice}

To test whether BDMPs aggravate and Lactadherin treatment reduces axonal/WM injury after ischemic stroke in mice, we employed MBP, BS, and LFB staining to quantify WM changes in the cortex and striatum of IBZ. Figure 3 shows that injection of BDMPs significantly decreases axon and myelin density in the IBZ compared to stroke group. Lactadherin treatment significantly increases axon and myelin density compared to stroke mice treated with PBS or BDMPs, respectively.

\section{BDMPs Significantly Increase and Lactadherin Treatment Significantly Decreases Neuroinflammation After Stroke in Mice}

To evaluate the inflammatory responses of BDMP injection and Lactadherin treatment in stroke mice, we measured the expression of leukocytes, microglia/macrophages, neutrophils, IL1 $\beta$, IL6, and TNF $\alpha$ in the cortex and striatum of IBZ. As indicated in Figures 4, 5, injection of BDMPs after 


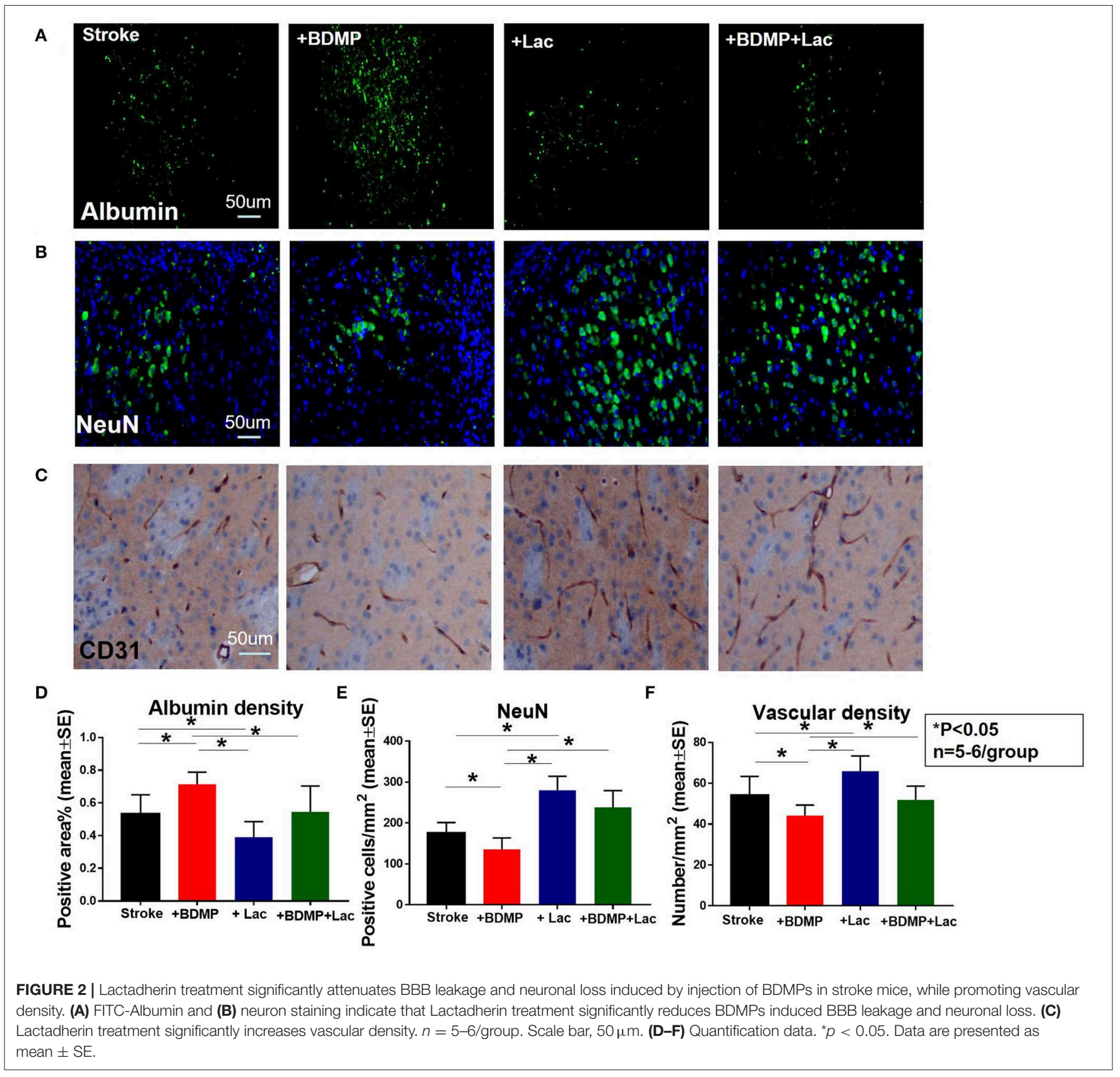

stroke significantly increases inflammatory cell and proinflammatory factor expression compared to stroke alone group. Lactadherin treatment significantly decreases inflammatory cell expression after stroke as well as attenuates BDMP induced neuroinflammation. In addition, Lactadherin treatment also significantly increases anti-inflammatory factor IL10 expression in IBZ compared to stroke mice treated with PBS or BDMPs, respectively (Figure 5). Figures 5I,J shows that BDMP injection significantly increases inflammatory factor IL1 $\beta$ expression in the circulation, while Lactadherin treatment significantly decreases IL1 $\beta$ expression in the circulation compared to stroke mice treated with PBS or BDMPs, respectively.

\section{DISCUSSION}

In this study, we demonstrate for the first time that BDMPs aggravate and Lactadherin attenuates stroke induced neurological deficits, BBB leakage, loss of vascular density, neuronal loss, axonal/WM injury and neuroinflammation after stroke in mice. These data suggest that neuroinflammation mediated by BDMPs may contribute to brain injury after stroke.

Extracellular MPs may play an important role in the pathological development and prognosis after stroke $(8,11,32)$. MPs can induce neuronal damage and neurotoxicity $(33,34)$. Previous studies have reported that the injured brain releases 


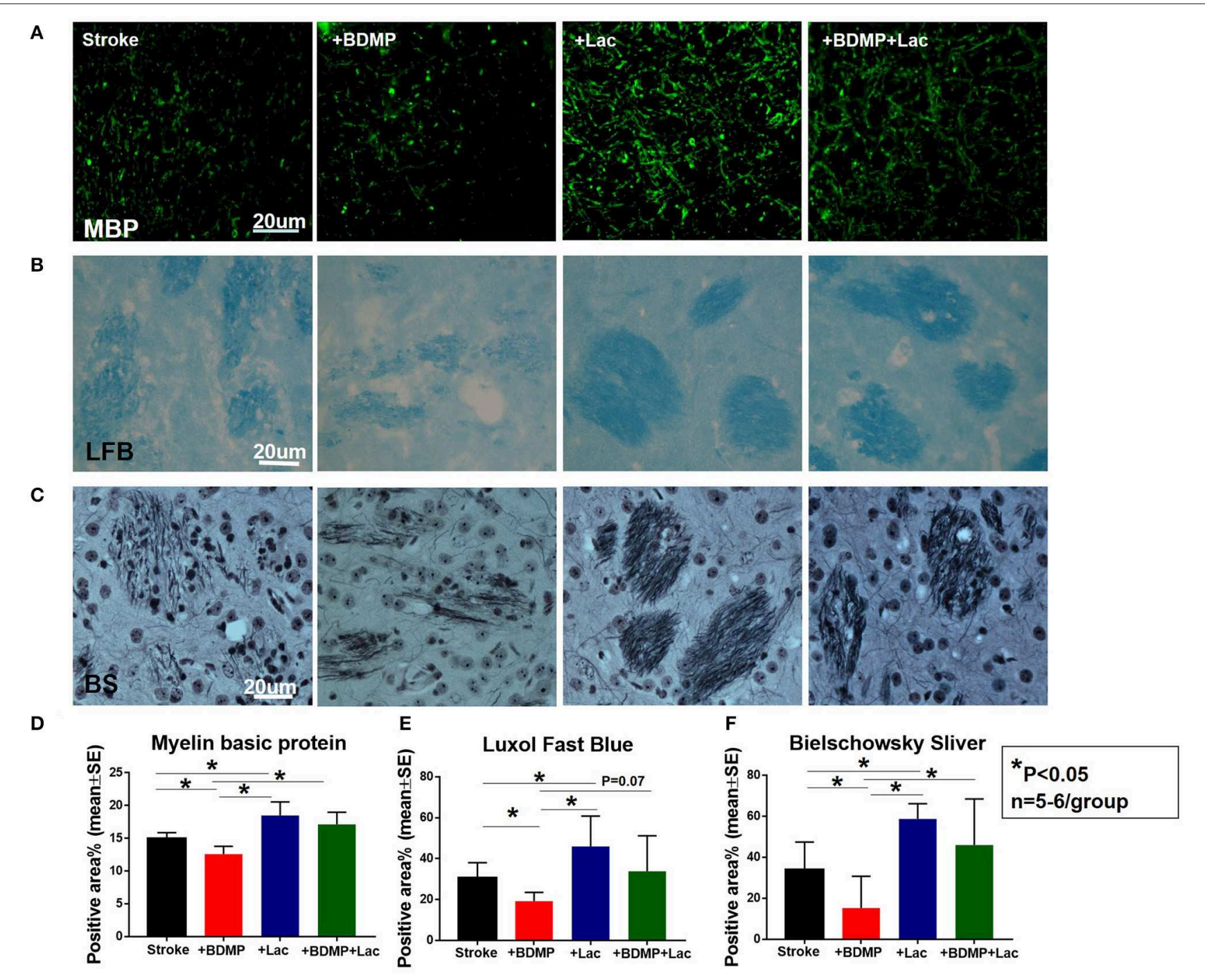

FIGURE 3 | Lactadherin treatment significantly attenuates axonalWM damage induced by BDMPs in stroke mice. (A) MBP, (B) Luxol fast blue, and (C) Bielschowsky sliver staining data indicate that Lactadherin treatment significantly increases axonal/WM density after injection of BDMPs in stroke mice, while BDMPs significantly increases axonal/WM damage. $n=5-6 /$ group. Scale bar, $20 \mu \mathrm{m}$. (D-F) Quantification data. ${ }^{*} p<0.05$. Data are presented as mean \pm SE.

BDMPs into the circulation $(5,7,9,16)$. Circulating MPs are lipid encased containers (sized $0.1-1.0 \mu \mathrm{m}$ ) that are shed from the plasma membrane of eukaryotic cells upon injury, activation, or apoptosis (35). Microparticles are vulnerable to degradation and clearance. Charoenviriyakul et al. reported that after intravenous injection different types of exosomes into mice, all the exosomes rapidly disappeared from the systemic circulation and were primarily localized to the liver (36). Exosomes can be taken up by macrophages and undergo clearance (37).Pharmacokinetic studies show that intravenously injected exosomes in mice were $10 \%$ of the initial injected amount at $4 \mathrm{~h}$ post injection (36). Clearance of all types of exosomes in macrophage-depleted mice was significantly delayed compared to that in non-macrophage depleted mice, indicating that macrophages play a key role in the clearance of exosomes from the blood circulation (36). The clearance of circulating microparticles involve direct receptor binding of liver or spleen phagocytes to phosphatidylserine or to opsonization proteins on the microparticles $(38,39)$. The routes of clearance microparticles by cells and organs include endocytosis (clathrin- and caveolin-dependent and lipid-raftmediated), micropinocytosis, phagocytosis and membrane fusion (40). In addition, the rates of microparticle degradation and clearance vary and depend on the ways that cells interact with their environment (41). The pooled concentrations of total MP, i.e., BDMPs, platelet-derived MPs, endothelial-derived MPs, leukocyte-derived MPs, erythrocyte-derived MPs, and monocytederived MPs are significantly increased in ischemic stroke patients compared to non-cerebrovascular disease controls, all of which are associated with poor clinical outcome $(7,11,42,43)$. BDMPs can contribute to the progression of neuroinflammatory 


\section{A}

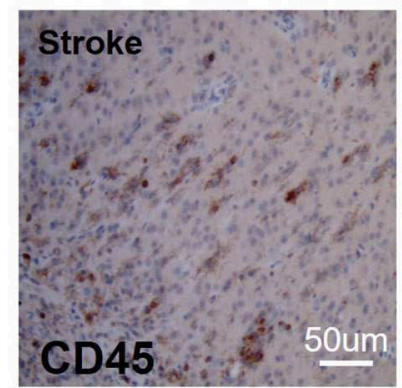

B

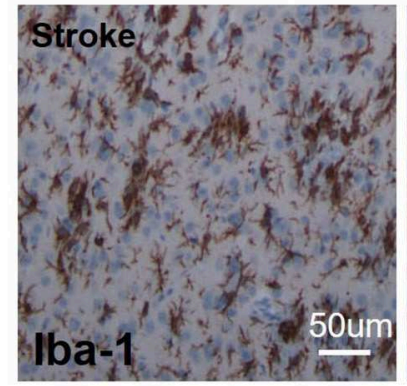

c

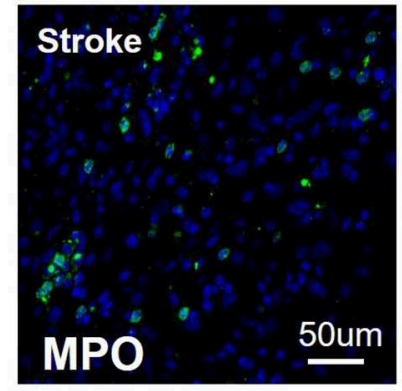

D

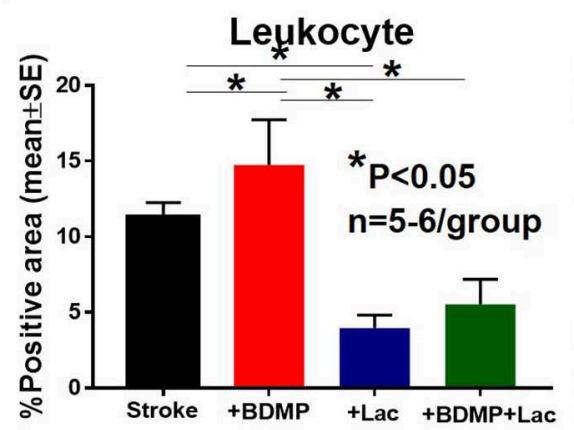

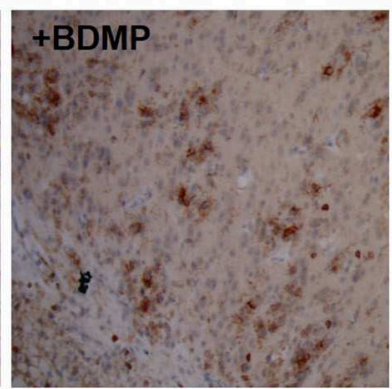
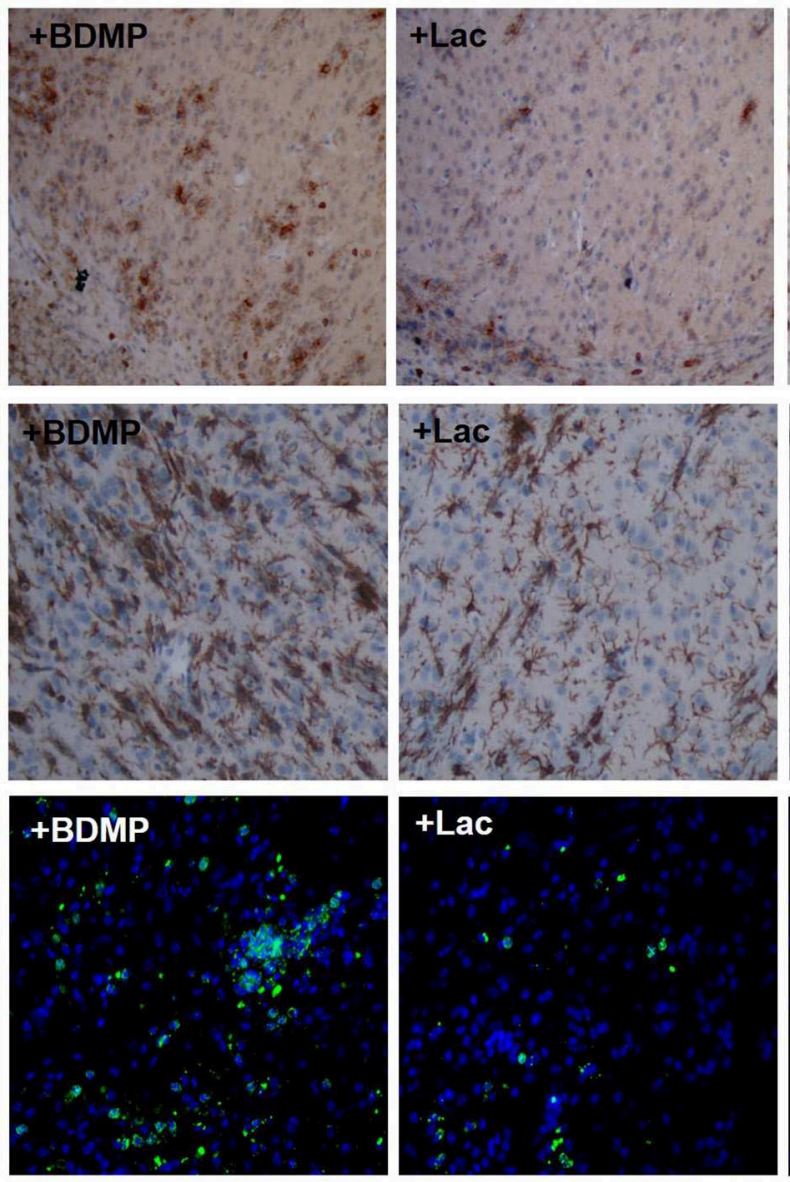
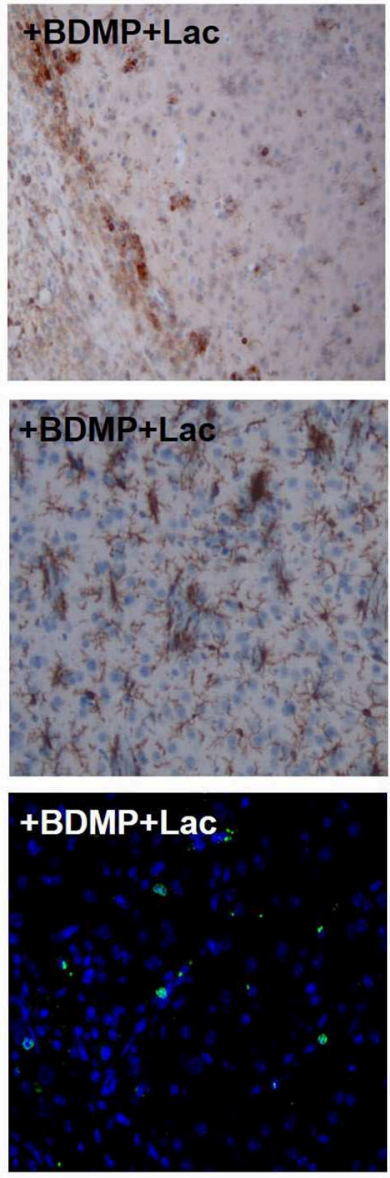

E

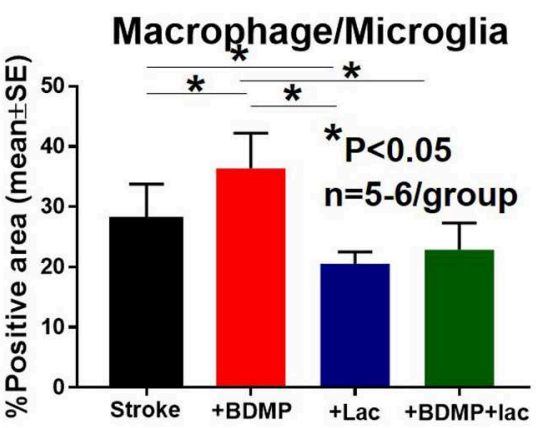

$\mathbf{F}$

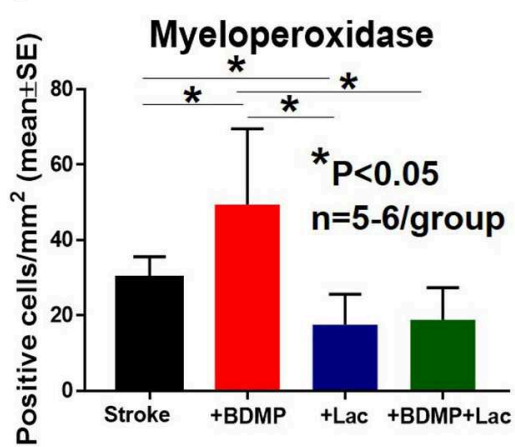

FIGURE 4 | BDMPs significantly increase and Lactadherin treatment significantly decreases inflammatory cell infiltration after stroke in mice. BDMPs significantly increase: (A) leukocyte (stained by CD45), (B) microglia/macrophage and, (C) neutrophil infiltration in ischemic brain, while Lactadherin treatment significantly decreases infiltration of inflammatory cell. $n=5-6 /$ group. Scale bar, $50 \mu \mathrm{m}$. (D-F) Quantification data. ${ }^{*} p<0.05$. Data are presented as mean \pm SE.

diseases and promote inflammatory activities, as well as promote the development and regeneration of the nervous system after stroke (44-46). Microglia-derived MPs and astrocyte-derived MPs contain and release the proinflammatory cytokine IL$1 \beta$, inflammasome components and MHCII proteins $(47,48)$. Previous studies have found that enriched MPs from activated microglia in vitro or from mice brain are sufficient to initiate neuroinflammation following intracortical injection in naïve animals $(16,17)$.

Lactadherin mediates cell-cell interactions and is involved in various physiological and pathophysiological functions including angiogenesis (49), fertilization (50), inflammation (23) and clearance of apoptotic cells (51). Several studies have confirmed the therapeutic effects of Lactadherin in stroke 


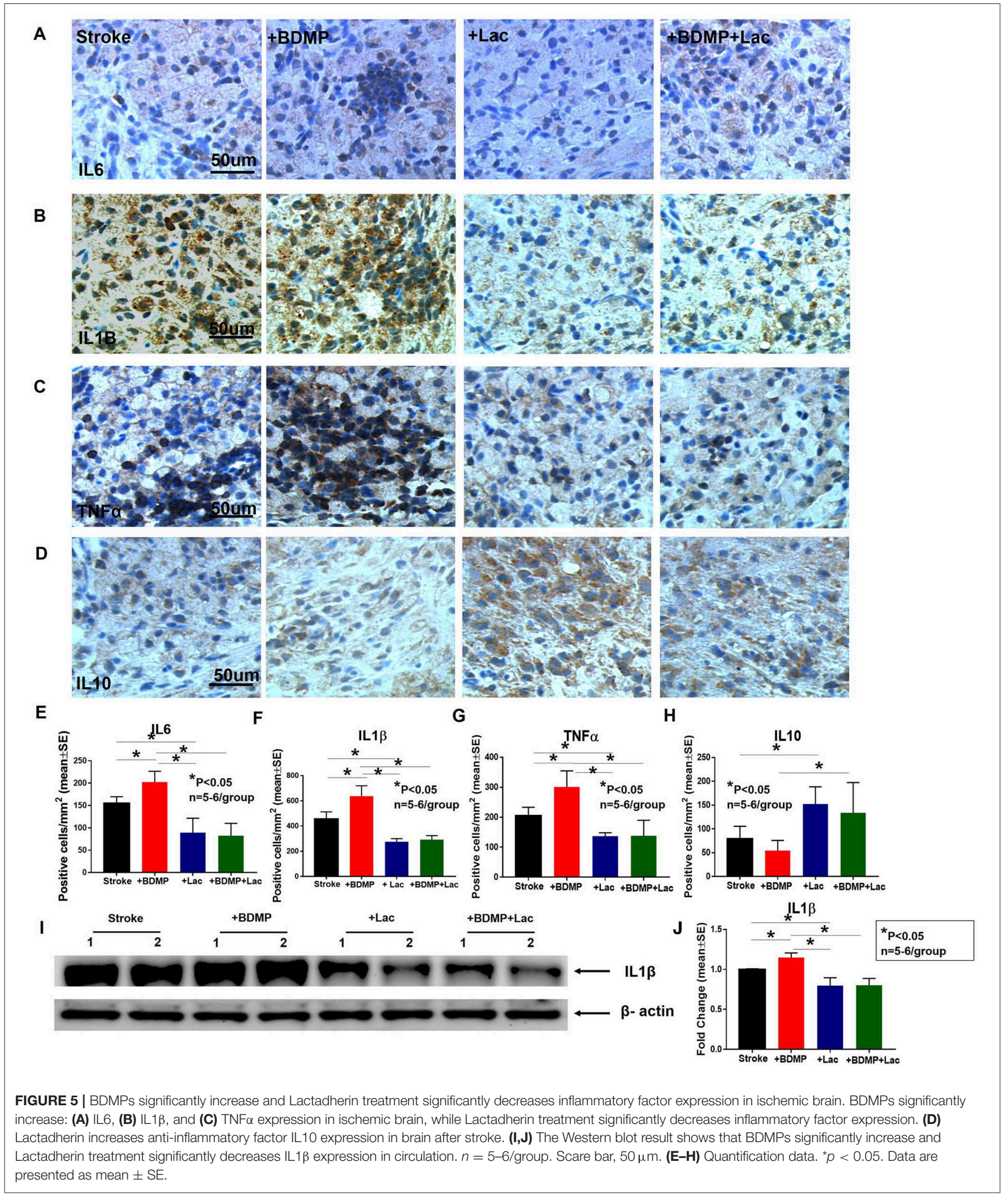

$(23,24,26,52)$. Lactadherin exerts neuroprotection against cerebral injury by suppressing inflammation, reducing neuronal cell death, promoting apoptotic cell clearance (26). Additionally,
Lactadherin improves subarachnoid hemorrhage (SAH) outcome via anti-oxidation which may be dependent on integrin $\beta 3 /$ nuclear factor erythroid 2-related factor 2/HO pathway 
(24). MFG-E8 maintains a role in the association between brain microvessels and surrounding brain parenchyma (53). A recent study indicates that Lactadherin can couple apoptotic cells with monocytes/macrophages to facilitate phagocytosis and promote the clearance of BDMPs $(19,21,22,54)$. We employed Lactadherin treatment together with BDMP administration to detect whether Lactadherin can promote neurological recovery by clearing BDMPs and decrease BDMP-induced neuroinflammation. Our data indicate that circulating BDMPs released by injured brain aggravate stroke outcome as evidenced by worse neurological deficits and exacerbated neuronal loss and lesion volume, while Lactadherin treatment improves neurological function, attenuates neuronal loss, and decreases lesion volume after stroke.

Physiological responses after stroke include BBB break down, delayed angiogenesis and neuron death (55). Previous studies have shown that neuronal damage in the central nervous system (CNS) is correlated with blood-brain barrier (BBB) breakdown (56-58). BBB is important for maintaining stability of the brain microenvironment of brain. BBB disruption permits infiltration of peripheral immune cells into the parenchyma and increases vascular oxidative stress and neuroinflammation which play a critical role in neuronal loss (59-61). MPs are known to increase $\mathrm{BBB}$ permeability $(18,62,63)$. Our data indicate that injection of BDMPs increase $\mathrm{BBB}$ leakage compared to the Stroke+PBS group, and Lactadherin treatment decreases BBB leakage which may contribute to improved neurological function in stroke mice. Post-ischemic angiogenesis has been widely associated with the recovery of blood flow in peri-infarct brain regions (64). The extent of angiogenesis plays a crucial role in long-term neurological function recovery (65-68). Our data show that injection of BDMPs reduces angiogenesis which may partially contribute to worse neurological function and increased infarction volume. Lactadherin treatment increases cerebral vascular density in the IBZ in stroke mice treated with PBS or BDMPs. Previous studies have demonstrated that MPs can induce neuronal damage and neurotoxicity $(33,34)$. Lactadherin can promote angiogenesis (49). Angiogenic vessels can increase cerebral blood flow in peri-infarcted brain regions, which may restore cellular metabolism in surviving neurons and promote neurogenesis (29). In our study, our data show that BDMPs increase neuronal loss in the IBZ, and Lactadherin treatment attenuates neuronal damage.

The white matter (WM) in the brain is highly susceptible to hypoxia and is injured following ischemic stroke (29). WM injury impairs neuronal connectivity and induces worse outcome after stroke $(69,70)$. WM promotes communication and sensory/motor reflex, which helps to restore lost nerve function and reduce symptoms of paralysis caused by stroke (29). Thus, WM function such as axonal regeneration and regrowth, axonal sprouting and remyelination in the peri-infarct region is critical for long term functional recovery (71). In this study, we found that injection of BDMPs after ischemic stroke decreases axon and myelin density in the IBZ while Lactadherin treatment significantly increases axon and myelin density in the IBZ of stroke mice treated with PBS and BDMPs, respectively.
Ischemic stroke is known to trigger complex systemic and local immune responses (72-74). However, the underlying mechanisms of post-stroke neuroinflammation are largely unclear (72-74). Neuroinflammation plays a critical role in WM damage, axonal degeneration and myelin breakdown (75). Microglia are activated within minutes after stroke onset and stimulate the production of inflammatory cytokines and promote leukocyte infiltration which exacerbate brain damage (76). Our data show that BDMPs increase the infiltration of leukocytes, microglia/macrophages and neutrophils, and the expression of immune factors IL1 $\beta$, IL6, and TNF- $\alpha$ in the IBZ of stroke mice. The infiltration of immune cells and molecules may indirectly or directly increase BBB permeability and promote tissue damage after stroke (77). Infiltrating neutrophils and microglia/macrophages promote BBB breakdown, WM damage, vascular damage and contribute to poor stroke outcome (12, 78, 79). Pro-inflammatory cytokines such as TNF $\alpha$, IL6, and IL1 $\beta$ induce BBB hyperpermeability (12) as well as induce WM injury (80-82). Lactadherin treatment significantly reduces systemic inflammatory response, decreases immune cell infiltration into the ischemic brain, decreases pro-inflammatory cytokine expression in the IBZ, and increases anti-inflammatory responses in stroke and BDMP treated stroke mice. Therefore, BDMPs may exert their adverse effects on stroke outcome by promoting inflammatory responses and Lactadherin treatment decreases BDMPs and regulates anti-inflammatory responses to improve stroke outcome in mice.

\section{LIMITATIONS}

In this study we have demonstrated that BDMPs induce neuroinflammation and aggravate neurological impairment after stroke, and Lactadherin treatment improves stroke outcome by promoting clearance of MPs as well as by exerting antiinflammatory effects. In this study, we selected MPs derived directly from the fresh brain tissue. Future studies are needed to test the effects of MPs derived from brain and their role in mediating neuroinflammation after stroke. The mechanisms of BDMP clearance by Lactadherin warrant investigation. Ischemic stroke can induce profound vascular, axon/WM damage. Vascular remodeling is a complex process that involves changes of structure and architecture of blood vessels via cell growth, death, migration, and degradation of the extracellular matrix (ECM) (83). Ischemic stroke induces axon/WM damage (84). In this study, we demonstrated Lactadherin regulation of vascular density and BBB leakage and axon/myelin density at 14 days after stroke. Additional studies on the temporal profiles of Lactadherin on neurovascular remodeling and neurological outcomes are warranted.

\section{CONCLUSIONS}

In this study, we found that BDMPs increase neuroinflammation and exacerbate brain damage after stroke in adult mice. Lactadherin exerts anti-inflammatory effects, improves the 
clearance of BDMPs, and may be a therapeutic strategy to reduce stroke and BDMP induced neurological dysfunction.

\section{DATA AVAILABILITY STATEMENT}

All relevant data is contained within the manuscript.

\section{ETHICS STATEMENT}

The animal study was reviewed and approved by Institutional Animal Care and Use Committee of Henry Ford Health System.

\section{AUTHOR CONTRIBUTIONS}

ZC performed experiments, analyzed data, and wrote the manuscript. MC was involved in experimental design and gave final approval of manuscript. PV performed experiments and wrote the manuscript. AZ, WL, FW, and JL-W performed

\section{REFERENCES}

1. Lopez AD, Mathers CD, Ezzati M, Jamison DT, Murray CJ. Global and regional burden of disease and risk factors, 2001: systematic analysis of population health data. Lancet. (2006) 367:1747-57. doi: 10.1016/S0140-6736(06)68770-9

2. Di Carlo A. Human and economic burden of stroke. Age Ageing. (2009) 38:4-5. doi: 10.1093/ageing/afn282

3. Hankey GJ. Stroke. Lancet. (2017) 389:641-54. doi: 10.1016/S0140-6736(16)30962-X

4. Zhao Z, Zhou Y, Tian Y, Li M, Dong JF, Zhang J. Cellular microparticles and pathophysiology of traumatic brain injury. Protein Cell. (2017) 8:801-10. doi: 10.1007/s13238-017-0414-6

5. Zhang J, Zhang F, Dong JF. Coagulopathy induced by traumatic brain injury: systemic manifestation of a localized injury. Blood. (2018) 131:2001-6. doi: 10.1182/blood-2017-11-784108

6. Morel N, Morel O, Petit L, Hugel B, Cochard JF, Freyssinet JM, et al. Generation of procoagulant microparticles in cerebrospinal fluid and peripheral blood after traumatic brain injury. J Trauma. (2008) 64:698-704. doi: 10.1097/TA.0b013e31816493ad

7. Tian Y, Salsbery B, Wang M, Yuan H, Yang J, Zhao Z, et al. Brain-derived microparticles induce systemic coagulation in a murine model of traumatic brain injury. Blood. (2015) 125:2151-9. doi: 10.1182/blood-2014-09-598805

8. Chiva-Blanch G, Suades R, Crespo J, Pena E, Padro T, Jimenez-Xarrie E, et al. Microparticle shedding from neural progenitor cells and vascular compartment cells is increased in ischemic stroke. PLoS ONE. (2016) 11:e0148176. doi: 10.1371/journal.pone.0148176

9. Zhao Z, Wang M, Tian Y, Hilton T, Salsbery B, Zhou EZ, et al. Cardiolipinmediated procoagulant activity of mitochondria contributes to traumatic brain injury-associated coagulopathy in mice. Blood. (2016) 127:2763-72. doi: 10.1182/blood-2015-12-688838

10. Nekludov M, Mobarrez F, Gryth D, Bellander BM, Wallen H. Formation of microparticles in the injured brain of patients with severe isolated traumatic brain injury. J Neurotrauma. (2014) 31:1927-33. doi: 10.1089/neu.2013.3168

11. Wang B, Cai W, Zhang Z, Zhang H, Tang K, Zhang Q, et al. Circulating microparticles in patients after ischemic stroke: a systematic review and metaanalysis. Rev Neurosci. (2018). doi: 10.1515/revneuro-2017-0105. [Epub ahead of print].

12. Jiang X, Andjelkovic AV, Zhu L, Yang T, Bennett MVL, Chen J, et al. Bloodbrain barrier dysfunction and recovery after ischemic stroke. Prog Neurobiol. (2018) 163-4:144-71. doi: 10.1016/j.pneurobio.2017.10.001

13. Mezouar S, Mege D, Darbousset R, Farge D, Debourdeau P, DignatGeorge F, et al. Involvement of platelet-derived microparticles in experiments. JC was involved in experimental design, wrote the manuscript, analyzed data, and gave final approval of manuscript.

\section{ACKNOWLEDGMENTS}

The authors wish to thank Qinge Lu and Sutapa Santra for their technical assistance.

\section{SUPPLEMENTARY MATERIAL}

The Supplementary Material for this article can be found online at: https://www.frontiersin.org/articles/10.3389/fimmu. 2019.02747/full\#supplementary-material

Supplementary Figure 1 | BDMPs do not induce brain damage in sham control mice. (A) mNSS and (B) Foot-fault test show that injection of BDMP into sham non-stroke (Sham+BDMP) mice did not induce neurological functional deficit. (B) Axon/white matter damage were not evident in brain tissue identified by BS (C) and LFB (D) staining in Sham+BDMP group when compared to sham control mice. Injection of BDMP did not induce leukocyte $(\mathbf{E})$ infiltration or microglia (F) activation.

tumor progression and thrombosis. Semin Oncol. (2014) 41:346-58. doi: 10.1053/j.seminoncol.2014.04.010

14. Tual-Chalot S, Gagnadoux F, Trzepizur W, Priou P, Andriantsitohaina $\mathrm{R}$, Martinez MC. Circulating microparticles from obstructive sleep apnea syndrome patients induce endothelin-mediated angiogenesis. Biochim Biophys Acta. (2014) 1842:202-7. doi: 10.1016/j.bbadis.2013.11.017

15. Williams MS, Rogers HL, Wang NY, Ziegelstein RC. Do plateletderived microparticles play a role in depression, inflammation, and acute coronary syndrome? Psychosomatics. (2014) 55:252-60. doi: 10.1016/j.psym.2013.09.004

16. Kumar A, Stoica BA, Loane DJ, Yang M, Abulwerdi G, Khan N, et al. Microglial-derived microparticles mediate neuroinflammation after traumatic brain injury. J Neuroinflamm. (2017) 14:47. doi: 10.1186/s12974-017-0819-4

17. Rong $\mathrm{H}$, Fan $\mathrm{Y}$, Yang $\mathrm{M}$, Zhang $\mathrm{B}$, Sun D, Zhao Z, et al. Brain-derived microparticles activate microglia/macrophages and induce neuroinflammation. Brain Res. (2018) 1694:104-10. doi: 10.1016/j.brainres.2018.05.015

18. Edrissi H, Schock SC, Hakim AM, Thompson CS. Microparticles generated during chronic cerebral ischemia increase the permeability of microvascular endothelial barriers in vitro. Brain Res. (2016) 1634:83-93. doi: 10.1016/j.brainres.2015.12.032

19. Zhou Y, Cai W, Zhao Z, Hilton T, Wang M, Yeon J, et al. Lactadherin promotes microvesicle clearance to prevent coagulopathy and improves survival of severe TBI mice. Blood. (2018) 131:563-72. doi: 10.1182/blood-2017-08-801738

20. Hanayama R, Tanaka M, Miwa K, Shinohara A, Iwamatsu A, Nagata S. Identification of a factor that links apoptotic cells to phagocytes. Nature. (2002) 417:182-7. doi: 10.1038/417182a

21. Hanayama R, Tanaka M, Miyasaka K, Aozasa K, Koike M, Uchiyama $\mathrm{Y}$, et al. Autoimmune disease and impaired uptake of apoptotic cells in MFG-E8-deficient mice. Science. (2004) 304:1147-50. doi: 10.1126/science. 1094359

22. Dasgupta SK, Thiagarajan P. The role of lactadherin in the phagocytosis of phosphatidylserine-expressing sickle red blood cells by macrophages. Haematologica. (2005) 90:1267-8.

23. Deroide N, Li X, Lerouet D, Van Vre E, Baker L, Harrison J, et al. MFGE8 inhibits inflammasome-induced IL-1beta production and limits postischemic cerebral injury. J Clin Invest. (2013) 123:1176-81. doi: 10.1172/JCI65167

24. Liu F, Hu Q, Li B, Manaenko A, Chen Y, Tang J, et al. Recombinant milk fat globule-EGF factor-8 reduces oxidative stress via integrin beta3/nuclear factor erythroid 2-related factor 2/heme oxygenase pathway in subarachnoid hemorrhage rats. Stroke. (2014) 45:3691-7. doi: 10.1161/STROKEAHA.114.006635 
25. Liu F, Chen Y, Hu Q, Li B, Tang J, He Y, et al. MFGE8/Integrin beta3 pathway alleviates apoptosis and inflammation in early brain injury after subarachnoid hemorrhage in rats. Exp Neurol. (2015) 272:120-7. doi: 10.1016/j.expneurol.2015.04.016

26. Gao YY, Zhang ZH, Zhuang Z, Lu Y, Wu LY, Ye ZN, et al. Recombinant milk fat globule-EGF factor-8 reduces apoptosis via integrin beta3/FAK/PI3K/AKT signaling pathway in rats after traumatic brain injury. Cell Death Dis. (2018) 9:845. doi: 10.1038/s41419-018-0939-5

27. Lee JK, Park MS, Kim YS, Moon KS, Joo SP, Kim TS, et al. Photochemically induced cerebral ischemia in a mouse model. Surg Neurol. (2007) 67:620-5; discussion: 625. doi: 10.1016/j.surneu.2006.08.077

28. Chen J, Sanberg PR, Li Y, Wang L, Lu M, Willing AE, et al. Intravenous administration of human umbilical cord blood reduces behavioral deficits after stroke in rats. Stroke. (2001) 32:2682-8. doi: 10.1161/hs1101.098367

29. Yan T, Venkat , Chopp M, Zacharek A, Ning R, Cui Y, et al. Neurorestorative therapy of stroke in type 2 diabetes mellitus rats treated with human umbilical cord blood cells. Stroke. (2015) 46:2599-606. doi: 10.1161/STROKEAHA.115.009870

30. Swanson RA, Morton MT, Tsao-Wu G, Savalos RA, Davidson C, Sharp FR. A semiautomated method for measuring brain infarct volume. J Cereb Blood Flow Metab. (1990) 10:290-3. doi: 10.1038/jcbfm.1990.47

31. Chen J, Zhang ZG, Li Y, Wang Y, Wang L, Jiang H, et al. Statins induce angiogenesis, neurogenesis, and synaptogenesis after stroke. Ann Neurol. (2003) 53:743-51. doi: 10.1002/ana.10555

32. He Z, Tang Y, Qin C. Increased circulating leukocyte-derived microparticles in ischemic cerebrovascular disease. Thromb Res. (2017) 154:19-25. doi: 10.1016/j.thromres.2017.03.025

33. Zhang R, Li Y, Hu B, Lu Z, Zhang J, Zhang X. Traceable nanoparticle delivery of small interfering RNA and retinoic acid with temporally release ability to control neural stem cell differentiation for Alzheimer's disease therapy. Adv Mater Weinheim. (2016) 28:6345-52. doi: 10.1002/adma.201600554

34. Guo J, Filpponen I, Johansson LS, Mohammadi P, Latikka M, Linder MB, et al. Complexes of magnetic nanoparticles with cellulose nanocrystals as regenerable, highly efficient, and selective platform for protein separation. Biomacromolecules. (2017) 18:898-905. doi: 10.1021/acs.biomac.6b01778

35. Giannella A, Radu CM, Franco L, Campello E, Simioni P, Avogaro A, et al. Circulating levels and characterization of microparticles in patients with different degrees of glucose tolerance. Cardiovasc Diabetol. (2017) 16:118. doi: 10.1186/s12933-017-0600-0

36. Charoenviriyakul C, Takahashi Y, Morishita M, Matsumoto A, Nishikawa M, Takakura Y. Cell type-specific and common characteristics of exosomes derived from mouse cell lines: yield, physicochemical properties, and pharmacokinetics. Eur J Pharm Sci. (2017) 96:316-22. doi: 10.1016/j.ejps.2016.10.009

37. Imai $T$, Takahashi $Y$, Nishikawa $M$, Kato $K$, Morishita M, Yamashita $T$, et al. Macrophage-dependent clearance of systemically administered B16BL6derived exosomes from the blood circulation in mice. J Extracell Vesicles. (2015) 4:26238. doi: 10.3402/jev.v4.26238

38. Flaumenhaft R. Formation and fate of platelet microparticles. Blood Cells $\mathrm{Mol}$ Dis. (2006) 36:182-7. doi: 10.1016/j.bcmd.2005.12.019

39. Davila M, Amirkhosravi A, Coll E, Desai H, Robles L, Colon J, et al. Tissue factor-bearing microparticles derived from tumor cells: impact on coagulation activation. J Thromb Haemost. (2008) 6:1517-24. doi: 10.1111/j.1538-7836.2008.02987.x

40. Mulcahy LA, Pink RC, Carter DR. Routes and mechanisms of extracellular vesicle uptake. J Extracell Vesicles. (2014) 3. doi: 10.3402/jev.v3.24641

41. Ayers L, Nieuwland R, Kohler M, Kraenkel N, Ferry B, Leeson P. Dynamic microvesicle release and clearance within the cardiovascular system: triggers and mechanisms. Clin Sci. (2015) 129:915-31. doi: 10.1042/CS 20140623

42. Huang $\mathrm{M}, \mathrm{Hu} \mathrm{YY}$, Dong XQ. High concentrations of procoagulant microparticles in the cerebrospinal fluid and peripheral blood of patients with acute basal ganglia hemorrhage are associated with poor outcome. Surg Neurol. (2009) 72:481-9; discussion: 489. doi: 10.1016/j.surneu.2008.12.016

43. Lackner P, Dietmann A, Beer R, Fischer M, Broessner G, Helbok $\mathrm{R}$, et al. Cellular microparticles as a marker for cerebral vasospasm in spontaneous subarachnoid hemorrhage. Stroke. (2010) 41:2353-7. doi: 10.1161/STROKEAHA.110.584995
44. Kumar A, Loane DJ. Neuroinflammation after traumatic brain injury: opportunities for therapeutic intervention. Brain Behav Immun. (2012) 26:1191-201. doi: 10.1016/j.bbi.2012.06.008

45. Obermeier B, Daneman R, Ransohoff RM. Development, maintenance and disruption of the blood-brain barrier. Nat Med. (2013) 19:1584-96. doi: $10.1038 / \mathrm{nm} .3407$

46. Porro C, Trotta T, Panaro MA. Microvesicles in the brain: biomarker, messenger or mediator? J Neuroimmunol. (2015) 288:70-8. doi: 10.1016/j.jneuroim.2015.09.006

47. Bianco F, Perrotta C, Novellino L, Francolini M, Riganti L, Menna E, et al. Acid sphingomyelinase activity triggers microparticle release from glial cells. EMBO J. (2009) 28:1043-54. doi: 10.1038/emboj.2009.45

48. Qu Y, Ramachandra L, Mohr S, Franchi L, Harding CV, Nunez G, et al. P2X7 receptor-stimulated secretion of $\mathrm{MHC}$ class II-containing exosomes requires the ASC/NLRP3 inflammasome but is independent of caspase-1. J Immunol. (2009) 182:5052-62. doi: 10.4049/jimmunol.0802968

49. Silvestre JS, Thery C, Hamard G, Boddaert J, Aguilar B, Delcayre A, et al. Lactadherin promotes VEGF-dependent neovascularization. Nat Med. (2005) 11:499-506. doi: 10.1038/nm1233

50. Ensslin MA, Shur BD. Identification of mouse sperm SED1, a bimotif EGF repeat and discoidin-domain protein involved in sperm-egg binding. Cell. (2003) 114:405-17. doi: 10.1016/S0092-8674(03)00643-3

51. Aziz M, Jacob A, Matsuda A, Wang P. Review: milk fat globule-EGF factor 8 expression, function and plausible signal transduction in resolving inflammation. Apoptosis. (2011) 16:1077-86. doi: 10.1007/s10495-011-0630-0

52. Cheyuo C, Jacob A, Wu R, Zhou M, Qi L, Dong W, et al. Recombinant human MFG-E8 attenuates cerebral ischemic injury: its role in antiinflammation and anti-apoptosis. Neuropharmacology. (2012) 62:890-900. doi: 10.1016/j.neuropharm.2011.09.018

53. Cheyuo C, Aziz M, Wang P. Neurogenesis in neurodegenerative diseases: role of MFG-E8. Front Neurosci. (2019) 13:569. doi: 10.3389/fnins.2019.00569

54. Dasgupta SK, Abdel-Monem H, Guchhait P, Nagata S, Thiagarajan P. Role of lactadherin in the clearance of phosphatidylserine-expressing red blood cells. Transfusion. (2008) 48:2370-6. doi: 10.1111/j.1537-2995.2008.01841.x

55. Yin KJ, Hamblin M, Chen YE. Angiogenesis-regulating microRNAs and ischemic stroke. Curr Vasc Pharmacol. (2015) 13:352-65. doi: 10.2174/15701611113119990016

56. Chen ZL, Indyk JA, Bugge TH, Kombrinck KW, Degen JL, Strickland S. Neuronal death and blood-brain barrier breakdown after excitotoxic injury are independent processes. J Neurosci. (1999) 19:9813-20. doi: 10.1523/JNEUROSCI.19-22-09813.1999

57. Cai W, Zhang K, Li P, Zhu L, Xu J, Yang B, et al. Dysfunction of the neurovascular unit in ischemic stroke and neurodegenerative diseases: an aging effect. Ageing Res Rev. (2017) 34:77-87. doi: 10.1016/j.arr.2016.09.006

58. Ohmori C, Sakai Y, Matano Y, Suzuki Y, Umemura K, Nagai N. Increase in blood-brain barrier permeability does not directly induce neuronal death but may accelerate ischemic neuronal damage. Exp Anim. (2018) 67:479-86. doi: 10.1538/expanim.18-0038

59. Varvel NH, Neher JJ, Bosch A, Wang W, Ransohoff RM, Miller RJ, et al. Infiltrating monocytes promote brain inflammation and exacerbate neuronal damage after status epilepticus. Proc Natl Acad Sci USA. (2016) 113:E56655674. doi: 10.1073/pnas.1604263113

60. Chen J, Wang Z, Zheng Z, Chen Y, Khor S, Shi K, et al. Neuron and microglia/macrophage-derived FGF10 activate neuronal FGFR2/PI3K/Akt signaling and inhibit microglia/macrophages TLR4/NF-kappaB-dependent neuroinflammation to improve functional recovery after spinal cord injury. Cell Death Dis. (2017) 8:e3090. doi: 10.1038/cddis.2017.490

61. Gelders G, Baekelandt V, Van der Perren A. Linking neuroinflammation and neurodegeneration in Parkinson's disease. J Immunol Res. (2018) 2018:4784268. doi: 10.1155/2018/4784268

62. Combes V, Coltel N, Faille D, Wassmer SC, Grau GE. Cerebral malaria: role of microparticles and platelets in alterations of the blood-brain barrier. Int J Parasitol. (2006) 36:541-6. doi: 10.1016/j.ijpara.2006.02.005

63. Lovren F, Verma S. Evolving role of microparticles in the pathophysiology of endothelial dysfunction. Clin Chem. (2013) 59:1166-74. doi: 10.1373/clinchem.2012.199711

64. Cui X, Chopp M, Zacharek A, Dai J, Zhang C, Yan T, et al. Combination treatment of stroke with sub-therapeutic doses of Simvastatin and 
human umbilical cord blood cells enhances vascular remodeling and improves functional outcome. Neuroscience. (2012) 227:223-31. doi: 10.1016/j.neuroscience.2012.09.066

65. Krupinski J, Kaluza J, Kumar P, Kumar S, Wang JM. Role of angiogenesis in patients with cerebral ischemic stroke. Stroke. (1994) 25:1794-8. doi: 10.1161/01.STR.25.9.1794

66. Marti HJ, Bernaudin M, Bellail A, Schoch H, Euler M, Petit E, et al. Hypoxia-induced vascular endothelial growth factor expression precedes neovascularization after cerebral ischemia. Am J Pathol. (2000) 156:965-76. doi: 10.1016/S0002-9440(10)64964-4

67. Zhang ZG, Zhang L, Tsang W, Soltanian-Zadeh H, Morris D, Zhang R, et al. Correlation of VEGF and angiopoietin expression with disruption of bloodbrain barrier and angiogenesis after focal cerebral ischemia. J Cereb Blood Flow Metab. (2002) 22:379-92. doi: 10.1097/00004647-200204000-00002

68. Ruan L, Wang B, ZhuGe Q, Jin K. Coupling of neurogenesis and angiogenesis after ischemic stroke. Brain Res. (2015) 1623:166-73. doi: 10.1016/j.brainres.2015.02.042

69. Schmidt RE. Neuropathology and pathogenesis of diabetic autonomic neuropathy. Int Rev Neurobiol. (2002) 50:257-92. doi: 10.1016/S0074-7742(02)50080-5

70. Walmsley AR, Mir AK. Targeting the Nogo-A signalling pathway to promote recovery following acute CNS injury. Curr Pharm Des. (2007) 13:2470-84. doi: 10.2174/138161207781368611

71. Singh B, Singh V, Krishnan A, Koshy K, Martinez JA, Cheng C, et al. Regeneration of diabetic axons is enhanced by selective knockdown of the PTEN gene. Brain. (2014) 137(Pt 4):1051-67. doi: 10.1093/brain/awu031

72. Barone FC, Feuerstein GZ. Inflammatory mediators and stroke: new opportunities for novel therapeutics. J Cereb Blood Flow Metab. (1999) 19:819-34. doi: 10.1097/00004647-199908000-00001

73. Samson Y, Lapergue B, Hosseini H. Inflammation and ischaemic stroke: current status and future perspectives. Rev Neurol. (2005) 161(12 Pt 1):117782. doi: 10.1016/S0035-3787(05)85190-2

74. Chamorro A, Hallenbeck J. The harms and benefits of inflammatory and immune responses in vascular disease. Stroke. (2006) 37:291-3. doi: 10.1161/01.STR.0000200561.69611.f8

75. Najjar S, Pearlman DM. Neuroinflammation and white matter pathology in schizophrenia: systematic review. Schizophr Res. (2015) 161:102-12. doi: 10.1016/j.schres.2014.04.041

76. Ning R, Venkat P, Chopp M, Zacharek A, Yan T, Cui X, et al. D-4F increases microRNA-124a and reduces neuroinflammation in diabetic stroke rats. Oncotarget. (2017) 8:95481-94. doi: 10.18632/oncotarget.20751
77. Gelderblom M, Leypoldt F, Steinbach K, Behrens D, Choe CU, Siler DA, et al. Temporal and spatial dynamics of cerebral immune cell accumulation in stroke. Stroke. (2009) 40:1849-57. doi: 10.1161/STROKEAHA.108. 534503

78. Moxon-Emre I, Schlichter LC. Evolution of inflammation and white matter injury in a model of transient focal ischemia. J Neuropathol Exp Neurol. (2010) 69:1-15. doi: 10.1097/NEN.0b013e3181c3ce6c

79. Zhang H, Zhang Y, Cao J, Zhou Y, Wang N, Zhou J. Determination of stage interconversion in vitro and in vivo by construction of transgenic Toxoplasma gondii that stably express stage-specific fluorescent proteins. Exp Parasitol. (2013) 134:275-80. doi: 10.1016/j.exppara.2013.03.015

80. Pang Y, Cai Z, Rhodes PG. Disturbance of oligodendrocyte development, hypomyelination and white matter injury in the neonatal rat brain after intracerebral injection of lipopolysaccharide. Brain Res Dev Brain Res. (2003) 140:205-14. doi: 10.1016/S0165-3806(02)00606-5

81. di Penta A, Moreno B, Reix S, Fernandez-Diez B, Villanueva M, Errea O, et al. Oxidative stress and proinflammatory cytokines contribute to demyelination and axonal damage in a cerebellar culture model of neuroinflammation. PLoS ONE. (2013) 8:e54722. doi: 10.1371/journal.pone.0054722

82. Fillman SG, Cloonan N, Catts VS, Miller LC, Wong J, McCrossin T, et al Increased inflammatory markers identified in the dorsolateral prefrontal cortex of individuals with schizophrenia. Mol Psychiatry. (2013) 18:206-14. doi: $10.1038 / \mathrm{mp} .2012 .110$

83. Mittal B, Mishra A, Srivastava A, Kumar S, Garg N. Matrix metalloproteinases in coronary artery disease. Adv Clin Chem. (2014) 64:1-72. doi: 10.1016/B978-0-12-800263-6.00001-X

84. Rosenzweig S, Carmichael ST. The axon-glia unit in white matter stroke: mechanisms of damage and recovery. Brain Res. (2015) 1623:123-34. doi: 10.1016/j.brainres.2015.02.019

Conflict of Interest: The authors declare that the research was conducted in the absence of any commercial or financial relationships that could be construed as a potential conflict of interest.

Copyright (c) 2019 Chen, Chopp, Zacharek, Li, Venkat, Wang, Landschoot-Ward and Chen. This is an open-access article distributed under the terms of the Creative Commons Attribution License (CC BY). The use, distribution or reproduction in other forums is permitted, provided the original author(s) and the copyright owner(s) are credited and that the original publication in this journal is cited, in accordance with accepted academic practice. No use, distribution or reproduction is permitted which does not comply with these terms. 